\title{
An Auto Tuning Substation Peak Shaving Controller for Congestion Management Using Flexible Demand
}

\author{
Sossan, Fabrizio; Marinelli, Mattia
}

Published in:

Proceedings of UPEC 2013

Publication date:

2013

Link back to DTU Orbit

Citation $(A P A)$ :

Sossan, F., \& Marinelli, M. (2013). An Auto Tuning Substation Peak Shaving Controller for Congestion Management Using Flexible Demand. In Proceedings of UPEC 2013 IEEE.

\section{General rights}

Copyright and moral rights for the publications made accessible in the public portal are retained by the authors and/or other copyright owners and it is a condition of accessing publications that users recognise and abide by the legal requirements associated with these rights.

- Users may download and print one copy of any publication from the public portal for the purpose of private study or research.

- You may not further distribute the material or use it for any profit-making activity or commercial gain

- You may freely distribute the URL identifying the publication in the public portal

If you believe that this document breaches copyright please contact us providing details, and we will remove access to the work immediately and investigate your claim. 


\title{
An Auto Tuning Substation Peak Shaving Controller for Congestion Management Using Flexible Demand
}

\author{
Fabrizio Sossan and Mattia Marinelli \\ Center for Electric Power and Energy at Technical University of Denmark \\ 4000 Roskilde, Denmark \\ ffaso,matm\}@elektro.dtu.dk
}

\begin{abstract}
A closed loop substation peak shaving/congestion management controller for radial distribution networks is presented. The controller it uses an individual control signal in order to shift the consumption of a population of demand side resources, DSRs. The controller auto tunes its parameters on-line using time series of the aggregate power consumption. No communication with the single DSRs is required. Simulations for validating the proposed control strategy using the MV CIGRE' reference network are presented.

Keywords - Power distribution, Smart grids, peak shaving, indirect control, Congestion Management
\end{abstract}

\section{INTRODUCTION}

Exploiting the flexibility at demand side level is becoming of increasing focus in order to supply power system services to the grid, and allowing to increase the share of production from renewables in the power system. The electric loads which exhibit flexibility in the electric power consumption are the ones with an intrinsic storage capability such as space heating and cooling units and refrigeration devices. This electric loads are referred with the term Demand Side Resources, DSRs, and their power consumption can be deferred according the needs of the power system as long as operational constraints and user comfort levels are respected. Two approaches are recognized for controlling DSRs: direct and indirect control. Direct control is the capability of controlling the consumption of each individual DSR [1], while indirect control simplifies the complex task of controlling a large population of DSRs by using an individual indirect control signal (e.g. the electricity price [2]) for all of them. Differently from direct control, indirect control relies on 1-way communication and it has less requirements in terms of communication infrastructure. In indirect control scheme, units are not required to communicate their flexibility. Therefore the potential support that DSRs could give to the power system has to be estimated [3].

In this paper a demand side management strategy for solving congestions in the distribution network is proposed. Congestion management has been extensively investigated in literature especially in connection with transmission lines connecting several portion of a power system [4]. In this context, control-by-price strategies have been proposed for rescheduling the production and removing congestions from overloaded segments (e.g. [5]). However these methods only applies to meshed networks and dispatchable production. Congestions in radial distribution networks have been mainly investigated with reference to smart charging algorithms in scenarios with high EVs penetration [6], [7], where bidirectional communication is used.

In this paper a closed loop controller for radial distribution networks is presented. The controller is placed in each substation of the network and it produces an individual control signal for indirect controlling the underneath flexible demand (electric space heating) with the aim of reducing a congestion whenever it arises. The controller is PI based and it auto tunes its parameters by estimating flexible demand sensitivity using time series measurements of the aggregate power consumption. The controller does not require bidirectional communication with the DSRs. A similar work, but on a smaller network and with static gains controller, has been proposed [8]. In the present work simulations for showing the proof of concept are realized using CIGRE' medium voltage reference network. Seven substations are considered in order to highlight the auto tuning capability of the controller. The population of indirect controlled DSRs are buildings heated by electric space heating. Simulations are performed with a co-simulation tool. The simulation setup together with the proposed congestion controller are discussed in Section II. Section III presents the results. Discussion of results is provided in Section IV. Conclusions are stated in Section V.

\section{MethodS}

Simulations are realized using the CIGRE' reference network (discussed in Section II-A), which is simulated in Matlab Simulink. Flexible demand of each substation of the network is simulated with a bottom up approach using a Python library, and it is presented in Section II-B. Flexible demand is represented inside each substation of the network by a variable load. The 
controller (discussed in Section II-C) is implemented in each substation of the network and, by detecting congestions, it shifts the consumption of flexible demand by producing a suitable real-time indirect control signal (which is sent to the Python engine that simulates flexible demand). Simulation sample time is $300 \mathrm{~s}$.

\section{A. Electric network layout}

The CIGRE' MV European reference network has been used as a case study [9]. The feeders of the reference network are three-phase and either of meshed or radial structure, with the latter dominating rural installations. Each feeder includes numerous laterals at which MV/LV transformers could be connected. The nominal voltage is $20 \mathrm{kV}$ and the system frequency is $50 \mathrm{~Hz}$. The study has been performed in the urban feeder which is shown in Fig. 1 and whose main information are here listed:

- $\quad 11$ MV buses and 11 LV (Low Voltage) buses;

- $4590 \mathrm{kVA}$ of transformer MV/LV capacity;

- $2.82 \mathrm{~km}$ OHL (OverHead lines) and $12.2 \mathrm{~km}$ Cable lines.

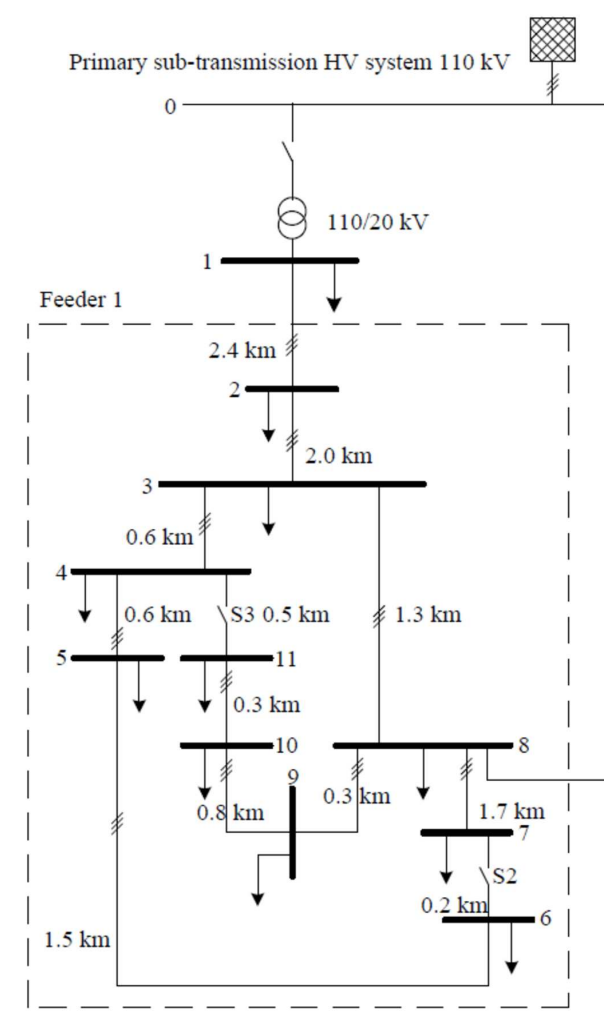

Fig. 1: Urban feeder of the CIGRE' network.

Two configuration switches (S2, S3) are present in Fig. 1 network but for this study are left in open status.
In the simulations here reported $7 \mathrm{MV} / \mathrm{LV}$ substations out of 11 possible are used for simulations. Low voltage network in the substations is not represented in details. Each substation is composed by 3 controllable loads:

- lumped flexible demand load;

- lumped residential base load;

- lumped commercial base load (not present in all substations);

The power consumption of the lumped flexible load is the aggregate power consumption of a subset of an indirect controlled population of DSRs. The number of DSRs for each subset depends on the nominal power of the respective substation transformer.

The residential and commercial lumped loads are subject to predefined daily consumption patterns defined by [9] and shown in Fig. 2. For showing the profof-concept of the proposed controller being able to clear congestions, the conventional consumption patterns have been amplified of a factor 1.3 in order to incur in congestions during peak hours. This could represent, for example, the effect that is provoked by an increase penetration of EVs.

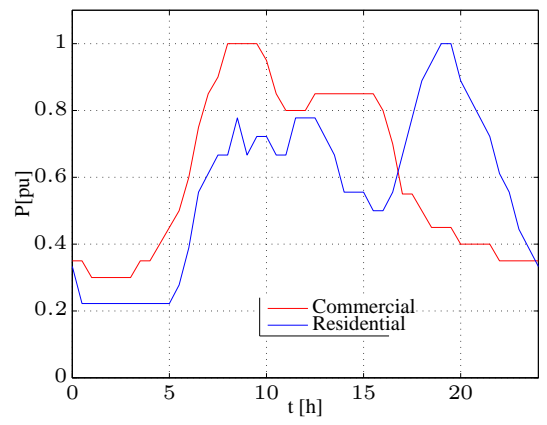

Fig. 2: Static daily patterns for the conventional residential and commercial consumption.

\section{B. DSRs population}

The indirect controllable demand side population is composed by a total of 346 buildings equipped with electric radiators for space heating. DSRs are divided in 7 groups (one for substation) whose sizes depend on the nominal power of the substation they are coupled to. The indoor temperature $T^{i n}\left[{ }^{\circ} \mathrm{C}\right]$ of each building is simulated using a second order linear dynamic thermal model. The parameters of each model are variated for accounting variations in building size and characteristics (see [10]). The nominal thermal model is given in Eq. 1 and Eq. 2, where $\mathbf{x} \in \mathbb{R}^{2 \times 1}$ is the vector state representing the thermal mass associated to air and building envelope, $P$ is the controllable building heating power $[\mathrm{kW}], T^{\text {out }}$ is the external temperature 
and $S$ is the solar irradiance [W] (these last two are not controllable inputs).

$$
\begin{aligned}
\frac{d \mathbf{x}}{d t} & =\left[\begin{array}{ccc}
-2.73 \times 10^{-5} & 9.15 \times 10^{-6} \\
1.48 \times 10^{-3} & -1.48 \times 10^{-3}
\end{array}\right] \mathbf{x} \\
& +\left[\begin{array}{ccc}
0 & 1.81 \times 10^{-5} & 7.38 \times 10^{-4} \\
1.48 \times 10^{-2} & 0 & 0
\end{array}\right]\left[\begin{array}{c}
P \\
T^{\text {out }} \\
S
\end{array}\right] \\
T^{i n} & =\left[\begin{array}{ll}
1 & 0
\end{array}\right] \mathbf{x}
\end{aligned}
$$

Model in Eq. 1 and Eq. 2 is from [11], and it has been realized applying grey-box modelling to Power Flexhouse, a DTU Elektro free standing building that is used for testing demand side strategies for domestic electric flexible demand.

DSRs indirect control algorithm: the power consumption of each building is indirect controlled by the controller described by Eq. 3, Eq. 4 and Eq. 5 which acts on the top a traditional thermostat. Such a controller which has been originally proposed in [2] for controlling TCLs (thermostatic controlled loads) using a real time electricity price. In Eq. $3, T_{i}^{\text {set-point }}$ is the thermostat set-point value at the discrete time step $i$, and it is calculated by adding an offset $\Delta T_{i}$ to the comfort indoor temperature $T^{o} . \Delta T_{i}$ is given in Eq. 4, where $k$ is an arbitrary coefficient for adjusting the sensitivity of the algorithm and $\hat{p}_{i}$ is the relative price, which is computed as given in Eq. 5, where $p_{i}$ is the current electricity price, $\bar{p}_{i}$ and $\sigma_{i}$ are the arithmetic mean and standard deviation of historical prices respectively, and they are calculated on a rolling window time series of arbitrary length ( $24 \mathrm{~h}$ in this case).

$$
\begin{aligned}
& T_{i}^{\text {set-point }}=T^{o}+\Delta T_{i} \\
& \Delta T_{i}=-k \hat{p}_{i} \\
& \hat{p}_{i}=\frac{p_{i}-\bar{p}_{i}}{\sigma_{i}} \quad \sigma_{i} \neq 0
\end{aligned}
$$

The standard deviation at the numerator of Eq. 5 acts as normalizing factor and it reduces the consumer risk of being exposed to high volatility of the price $p$. At steady state $(d p / d t=0)$, the relative price in Eq. 5 tends to zero as the price $p_{i}$ tends to match its average $\bar{p}_{i}$ and the term at numerator becomes 0 . Therefore, at steady state, the user comfort is not affected.

In the context of this work, no price signal is used and $p$ is the indirect control signal produced by the substation controller.

\section{Congestion controller}

The discrete control loop implemented in each substation of Fig. 1 network is shown in Fig. 3. The term $P_{e_{i}}$ is the measured power transit [pu] at the transformer, that is composed both by conventional and flexible demand. The input of the controller is given by $P_{e_{i}}-1$, where $1 \mathrm{pu}$ is the nominal power of the transformer. The output of the controller is the indirect control signal $p$ that is broadcasted to all the DSRs belonging to that substation. Subscripts $i$ refer to discrete time instant.

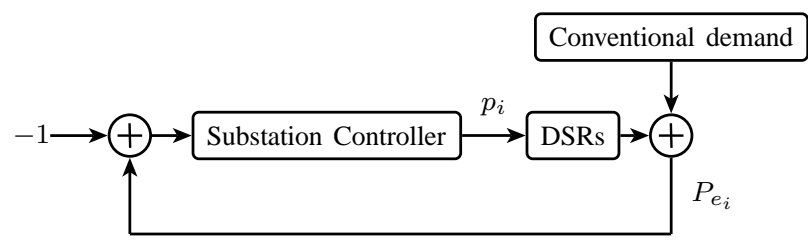

Fig. 3: Feedback control loop implemented in each substation.

Fig. 4 shows the structure of the substation controller. ${ }^{1}$ The core of the controller is a PI regulator with gains proportional to $s_{m}$, which is an adaptive parameter discussed in the next section. The two saturation blocks on the input side of the diagram are respectively for feeding the controller only when a power transit overload occurs (upper block) and for allowing to remove the integral action when the congestion is solved (lower block). The integral regulator is equipped with an antiwindup configuration (for not accumulating an error when congestions are not occurring) which is not shown in the diagram.

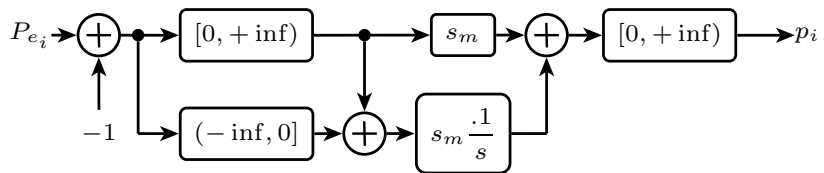

Fig. 4: The proposed substation controller for congestion management.

\section{Determination of coefficient $s_{m}$}

Coefficient $s_{i}$ is defined as the value of the variation of the indirect control signal $\Delta p_{i}=p_{i}-p_{i-1}$ that induces a unitary change of the aggregate power consumption $\Delta P_{i}=P_{i}-P_{i-1}[\mathrm{~kW}]$, and it is given in Eq. 6.

$$
s_{i}=\frac{\Delta p_{i}}{\Delta P_{e_{i}}}, \Delta P_{e_{i}} \neq 0
$$

Assuming to know $s_{i}$ at each instant of time $i$, the deviation of the indirect control signal that should be applied for changing the aggregate power consumption of a generic amount $x$ could be computed as given in Eq. 7.

$$
\Delta p_{i}=s_{i} \cdot x
$$

\footnotetext{
${ }^{1}$ Integrators are indicated with $\frac{1}{s}$ for being compact. However their discretized version is meant.
} 
By knowing the amount of overload at a given substation, Eq. 7 could be used for finding the indirect control signal deviation to apply for clearing the congestion. However, $s_{i}$ is not known, and besides, it is a time varying quantity and a function of DSRs conditions, for example DSRs state, consumers comfort.

The solution that is adopted in the proposed controller consists of observing the quantities $\Delta P_{e_{i}}$ and $\Delta p_{i}$ at each instant of time and evaluating the coefficient $s_{m}$ as given in Eq. 7.

$$
s_{m}=\min \left\{s_{i}\right\}
$$

The term $s_{m}$ is the the gain of both proportional and integral part (scaled) of the regulator. The task of the integral action is increasing the indirect control signal if DSRs are not decreasing consumption enough: in fact, $s_{m}$ might overestimates the sensitivity of the flexible demand to variation of the indirect control signal. Computing the value in Eq. 6 requires the know the value of the aggregate power consumption of DSRs. However, since the variation of power to a given variation of the control signal is of interest, the measure of the power transit at the substation transformer is used because conventional demand does not react to the control signal, hence its contribution does not affect Eq. 6. Bidirectional communication with DSRs is hence not required. The adaptive method discussed in this section is implemented in all substation congestion controllers. The controllers do not hence require any manual intervention for adjusting the gains (cfr. [8]). An initial training phase is required for discovering the coefficient $s_{m}$ through Eq. 8. During operation, $s_{m}$ is updated on-line, and it is scaled by a forgetting factor in order to allow $s_{m}$ to be updated with recent values.

\section{RESULTS}

Simulations are performed using the urban feeder of the medium voltage CIGRE' reference network. Electrical demand is composed by a mix of conventional and flexible consumption. Each substation is equipped with an auto tuning feedback controller whose task is to send an individual control signal to all substation DSRs in order to shift their power consumption every time a congestion occurs. The loop is closed by measuring the power transit, i.e. no 2 -way communication is required with the DSRs. Two simulation scenarios are proposed. Scenario $a$ is with no congestion control while, in Scenario $b$, all substation controllers are activated. Both scenarios present same network configuration, conditions and consumption profiles. Simulations last for 24 hours, starting from midnight. The time series used for evolving thermal models of buildings (outdoor temperature and solar radiation are from real measurements). Coherently with the notation used before, the power transits in the plots are expressed in pu, that is transit power divided the nominal capacity of the substation transformer. The power transit of each substation (from substation 1 to 7 ) of scenario $a$ are shown in Fig. 5 (no congestion management). The thick black line corresponds to $1 \mathrm{pu}$.

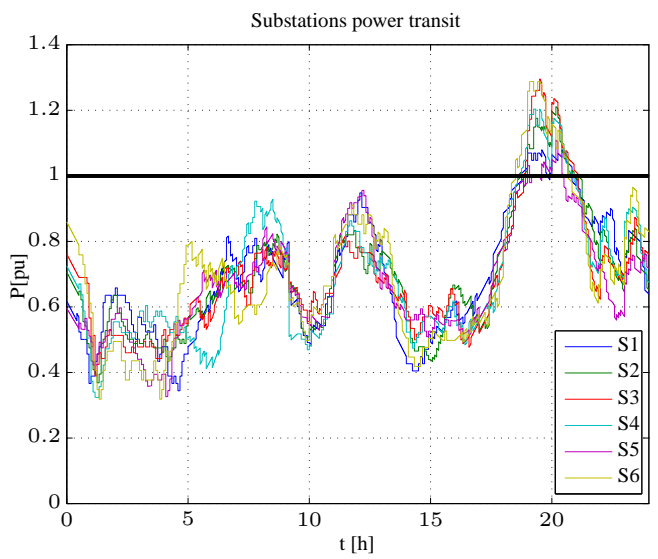

Fig. 5: Scenario $a$. Congestions are occurring in all the substations in the evening hours.

Substations power transits for Scenario b are shown in Fig. 6. Substation 0 is not shown in the figures because the power transit never exceed the nominal value.

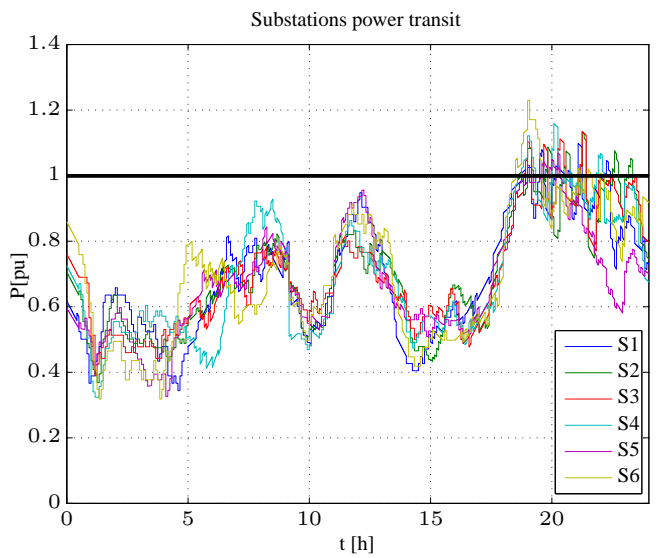

Fig. 6: Scenario $b$. Congestion controllers are activated in each substation and the consumption is trimmed thanks to acting on flexible demand.

\section{DISCUSSION}

Scenario $a$ in Fig. 5 depicts a situation where all the substations are congested during evening hours. Fig. 6 shows that in scenario $b$, the substations controllers starts to shift the consumption as soon as they detect 
an overload; consequently the DSRs tend to reduce their power consumption. A closer inspection to the power profiles of the different substations in Fig. 6, during the time frame where the congestions occur, shows that 1) the power consumption oscillates around $1 \mathrm{pu}$ or 2) it slowly decreases to one, 3) the peak values are reduced but the wave of high consumption last longer than scenario $b$. Case 1) occurs when $s_{m}$ underestimates indirect control capability. In this case the flexible demand is very reactive to indirect control signal variations and the initial control action is mainly given by the proportional regulator, which increases and decreases the indirect control signal value according if the power transit is exceeding the nominal power transit or not. In case 2), the value $s_{m}$ overestimates indirect control capability. The integral action of the controller increases with time and causes the aggregate power consumption of the loads to decrease.

Case 3 ) is because the controller achieves consumption shifting, hence the demand is postponed. It is worth to mention that at this point the DSRs consumption is synchronized, causing the aggregate power consumption to oscillate for a period [10]. Table I reports some relevant information of the power transits of each substation. Data are referred to the time frame $\approx 18.5 \mathrm{~h}<t<\approx$ $20.7 \mathrm{~h}$ where congestion is occurring. The controller is able to trim the consumption peak values in all substations and in particular the mean values of the power transits are kept very close to the nominal ones. In this simulation, the proposed controller is hence able to reduce the congestions in all the substations meaning also that the auto tuning procedure here presented is well performing.

TABLE I: Substations power transit values during congestion

\begin{tabular}{cccccccc} 
Power transit & Scenario & S1 & S2 & S3 & S4 & S5 & S6 \\
\hline \hline \multirow{2}{*}{ peak value } & $b$ & 1.08 & 1.21 & 1.30 & 1.20 & 1.11 & 1.29 \\
& $c$ & 1.08 & 1.09 & 1.11 & 1.16 & 1.07 & 1.23 \\
\hline \multirow{2}{*}{ mean } & $b$ & 1.05 & 1.09 & 1.15 & 1.11 & 1.02 & 1.16 \\
& $c$ & 0.98 & 0.98 & 0.98 & 1. & 1.01 & 1.02 \\
\hline
\end{tabular}

\section{CONCLUSION}

A substation feedback controller for flexible demand to peak shave and reduce congestions in radial distribution networks is presented. The controller auto tunes its parameters on-line using measurements of the aggregate power consumption and it does not need 2way communication DSRs. In order to show the proof of concept, the performance of the controller have been tested using seven substations of the urban feeder of the medium voltage CIGRE' reference network. flexible demand is implemented by buildings equipped with electric space heating. Simulations showed that the proposed controller is able to shift the consumption when a congestion occurred. Besides, the tuning procedure is effective as performances were equally good in all the seven substations.

\section{REFERENCES}

[1] F. Bliek, A. van den Noort, B. Roossien, R. Kamphuis, J. de Wit, J. van der Velde, and M. Eijgelaar, "Powermatching city, a living lab smart grid demonstration," in Innovative Smart Grid Technologies Conference Europe (ISGT Europe), 2010 IEEE PES, pp. $1-8$, oct. 2010.

[2] D. J. Hammerstrom, P. Investigator, R. Ambrosio, T. A. Carlon, J. G. Desteese, R. Kajfasz, and R. G. Pratt, "Pacific Northwest GridWise Testbed Demonstration Projects Part I . Olympic Peninsula Project," Contract, p. 157, 2007.

[3] F. Sossan and H. Bindner, "Evaluation of the performance of indirect control of many dsrs using hardware-in-the-loop simulations," in CDC, 2012 Maui, HI, US, December 2012.

[4] T. Krause and G. Andersson, "Evaluating congestion management schemes in liberalized electricity markets using an agent-based simulator,' in Power Engineering Society General Meeting, 2006. IEEE, pp. 8-pp, IEEE, 2006.

[5] H. Glavitsch and F. Alvarado, "Management of multiple congested conditions in unbundled operation of a power system," in Power Industry Computer Applications., 1997. 20th International Conference on, pp. 374-380, 1997.

[6] J. Hu, S. You, M. Lind, and J. Østergaard, "Coordinated charging of electric vehicles for congestion prevention in the distribution grid," IEEE Transactions on Smart Grid, 2012.

[7] O. Sundstrom and C. Binding, "Flexible charging optimization for electric vehicles considering distribution grid constraints," Smart Grid, IEEE Transactions on, vol. 3, no. 1, pp. 26-37, 2012.

[8] Z. Csetvei, J. Ostergaard, and P. Nyeng, "Controlling priceresponsive heat pumps for overload elimination in distribution systems," in Innovative Smart Grid Technologies (ISGT Europe), 2011 2nd IEEE PES International Conference and Exhibition on, pp. 1-8, IEEE, 2011.

[9] C. T. F. C6.04.02, "Benchmark systems for network integration of renewable and distributed energy resources," tech. rep., Cigre' Internationl Council on large electric systems, July 2009.

[10] F. Sossan and all, "Dynamic behaviour of a population of controlled-by-price demand side resources," in Manuscript in preparation, pp. 1-5, 2014.

[11] H. M. P. Bacher, A. Thavlov, "Models for energy performance analysis," tech. rep., DTU - IMM, 2010. 